\title{
The ACE2-Ang (1-7)-Mas receptor axis attenuates cardiac remodeling and fibrosis in post-myocardial infarction
}

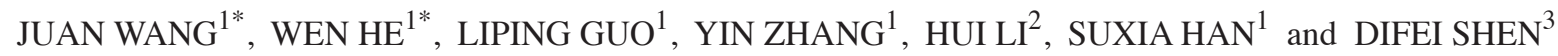 \\ ${ }^{1}$ Department of Cardiology, The Fifth Affiliated Hospital of Xinjiang Medical University; \\ ${ }^{2}$ Department of Cardiology, The First Affiliated Hospital of Xinjiang Medical University, Urumqi, Xinjiang 830001; \\ ${ }^{3}$ Department of Cardiology, Renmin Hospital of Wuhan University, Wuhan, Hubei 430060, P.R. China
}

Received June 23, 2016; Accepted April 19, 2017

DOI: $10.3892 / \mathrm{mmr} .2017 .6848$

\begin{abstract}
Myocardial remodeling serves an important role in the pathophysiology of coronary heart disease. The angiotensin-converting enzyme (ACE)2-angiotensin-(1-7) [Ang (1-7)]-Mas receptor (MasR) axis is a key regulator in myocardial remodeling and development of heart failure. To investigate how ACE2-Ang-(1-7)-MasR axis function on myocardial remodeling and cardiac fibrosis in post-myocardial infarction (MI), male Sprague-Dawley rats (weight, 200 $\pm 20 \mathrm{~g}$ ) were used to establish the model of myocardial infarction by ligating the left coronary artery. The present study suggests that telmisartan (Tel) and olmesartan $(\mathrm{Olm})(5 \mathrm{mg} / \mathrm{kg} / \mathrm{d})$ can inhibit myocardial remodeling of post-myocardial infarction through the ACE2-Ang (1-7)-MasR pathway. Administration of Tel or Olm was demonstrated to significantly inhibit collagen deposition using Masson staining. In addition, telmisartan and olmesartan was indicated to antagonize angiotensin II (Ang II) and upregulate ACE2, MasR, Ang (1-7) expression in myocardial tissue using immunoassay and ELISA test, and the effect of Olm was more marked than that of Tel at the same dosage. Simultaneously, compared with the MI or Sham group, the mRNA and protein expression of ACE2, Ang II and MasR in myocardial tissue demonstrated a remarkable increase in the Olm group, when compared with the Tel group. Taken together, our data demonstrated that ACE2-Ang (1-7)-MasR axis may present a potential protective role in the development
\end{abstract}

Correspondence to: Dr Suxia Han, Department of Cardiology, The Fifth Affiliated Hospital of Xinjiang Medical University, 118 Henanxi Road, Xinshi, Urumqi, Xinjiang 830001, P.R. China E-mail: hsx0016@163.com

Dr Difei Shen, Department of Cardiology, Renmin Hospital of Wuhan University, 99 Zhangzhidong Road, Wuchang, Wuhan, Hubei 430060, P.R. China

E-mail: 15827276696@163.com

\section{${ }^{*}$ Contributed equally}

Key words: angiotensin-converting enzyme 2, angiotensin-(1-7), Mas receptor, fibrosis, myocardial infarction, myocardial remodeling of myocardial remodeling and may provide a new target for drug development of cardiac fibrosis. In conclusion, $\mathrm{Olm}$ is superior to Tel in inhibiting myocardial local Ang II level reducing myocardial collagen deposition and improving myocardial remodeling by upregulating the expression of ACE2, Ang (1-7) and MasR.

\section{Introduction}

Cardiovascular disease (CVD) is the leading cause of mortality worldwide and according to the 2014 statistics, an estimated 17.5 million non-communicable disease deaths have been caused by CVD $(1,2)$. Myocardial remodeling in post-myocardial infarction is one of the pathophysiological bases of CVD and eventually results in many serious complications. Myocardial remodeling refers to changes in myocardial structure, function and phenotype caused by a series of complex molecular and cellular mechanisms. The renin-angiotensin system (RAS) is an important mediator of atherosclerosis, hypertension (HT), myocardial fibrosis, left ventricular (LV) hypertrophy, myocardial infarction and congestive heart failure (3-5).

The classicRAScanbedefined as the angiotensin-converting enzyme (ACE)-Ang II-AT1R axis that promotes vasoconstriction, water intake, sodium retention and other mechanisms, as well as increase oxidative stress, fibrosis, cellular growth and inflammation in pathological conditions (6). Numerous studies have indicated that the noncanonical RAS pathway ACE2-angiotensin-(1-7) [Ang (1-7)]-Mas receptor (MasR) axis counteracts the adverse effects of the classical pathway (6-8). ACE2 is a pleiotropic mono-carboxy peptidase, and belongs to the family of zinc metalloproteases, that can be found in a soluble form in plasma and tissues ubiquitously (9). ACE2 is involved in the structural and functional regulation of human heart. ACE2 overexpression significantly prevents myocardial fibrosis, reduces the development of LV hypertrophy (LVH) and improves cardiac function (10-13). Ang (1-7), one of the major enzymatic products of ACE2, can reduce Ang II-induced cardiac hypertrophy and remodeling and pressure-overload-induced heart failure $(6,7,14)$. The cardioprotective effects of Ang (1-7) are mediated by MasR through different signaling pathways $(15,16)$. Therefore, the ACE2-Ang (1-7)-MasR axis, known as the second metabolic axis of RAS, serves a pivotal role in cardiovascular disease. 
To elaborate the expression of the ACE2-Ang (1-7)-Mas axis in animals of ventricular remodeling in post-MI, the authors established a rat model of myocardial infarction. The expression of Ang II remarkably increased following development of myocardial infarction. In addition, Tel and Olm inhibited local level of myocardial Ang II, reduced myocardial collagen deposition and improved myocardial remodeling by upregulating the expression of ACE2, Ang (1-7) and MasR. The effect of Olm was greater that the effect of Tel. Thus, the ACE2-Ang (1-7)-Mas axis serve an important role in the development of ventricular remodeling in post-MI, which may provide a new target for CVD drug design and therapeutic strategy.

\section{Materials and methods}

Experimental animals and treatment. A total of 40 male Sprague-Dawley (SD) rats (weight 200 20 g, 2-months-old) were purchased from the Experimental Animal Center of Xinjiang Medicine University (Urumqi, China). Experimental protocols for animal studies were performed based on the 'Guide for the Care and Use of Laboratory Animals' and have been approved by the Institutional Animal Care and Use Committee at the Xinjiang Medicine University (Ethical review number: IACUC-20121127010). Male SD rats had free access to food and water, and were maintained in a temperature $\left(25^{\circ} \mathrm{C}\right)$ controlled room with a 12-h dark/light cycle. Male SD rats were randomly divided into a sham group $(n=10)$, a myocardial infarction $(\mathrm{MI})$ group $(\mathrm{n}=10)$, Tel group $(5 \mathrm{mg} / \mathrm{kg} / \mathrm{d} ; \mathrm{n}=10)$ and Olm group $(5 \mathrm{mg} / \mathrm{kg} / \mathrm{d} ; \mathrm{n}=10)$. In the Tel and Olm groups, intragastric administration of Tel and Olm were performed daily (15:00-16:00 pm) for 4 weeks following surgery. All rats in which the induction of remodeling had been successful were identified in each group.

The establishment myocardial infarction model. Rats were fasted for $6 \mathrm{~h}$ before the operation, and the rats' body weight was measured. Following full anesthesia through the intraperitoneal injection of $10 \%$ chloral hydrate $(100-200 \mathrm{mg} / \mathrm{kg})$, intubated and placed on a standard rodent ventilator. The adequacy of anesthesia was verified using tail pinch. An incision was made between the 3rd and 4th intercostal space, and a rib spreader was used to allow visualization of the heart. A 7-0 suture was used to ligate the left coronary artery at a location approximately 1-2 $\mathrm{mm}$ distal to the left atrium. Successful occlusion of the left anterior descending branch (LAD) was confirmed by elevation of the ST segment on the electrocardiogram and cyanosis of anterior LV wall. Immediately before or after surgery, morphine $(0.1 \mathrm{mg} / \mathrm{kg})$ was administered intraperitoneally to reduce pain and penicillin $\left(4 \times 10^{4} \mathrm{U} / \mathrm{rat}\right)$ was administered intramuscularly for three days to prevent infection. The mortality of the animals operated on in this fashion was $33.7 \%$ within $24 \mathrm{~h}$.

The echocardiographic examination. Transthoracic echocardiography was performed noninvasively to assess systolic and diastolic function, as described previously using a Doppler ultrasound instrument with a $12-\mathrm{Hz}$ transducer (Philips 21380A Ultra Band XDCR S12) M-mode echocardiography was performed in the parasternal short-axis view at the level of the papillary muscles. Mice were placed on a heating pad and a nose cone with $1.5 \%$ isoflurane in $100 \%$ oxygen was applied. Ultrasound gel was placed on the chest of the anesthetized mouse. End-diastolic interventricular septal thickness, end-diastolic left ventricular posterior wall thickness, left ventricular end-diastolic diameter, left ventricular ejection fraction were measured and recorded. All measurements were based on the average of three consecutive cardiac cycles.

Histological analysis. Left ventricular myocardial specimens were taken from each group and sectioned transversely parallel to the coronary sulcus. Masson staining was used to observe the degree of myocardial fibrosis; myocardial cells were red and collagen was blue. Five sections were selected for each specimen, and four views were taken for each section (the selected views were away from the papillary muscle and perivascular collagen). The Image-Pro Plus software (Media Cybernetics, Inc., Rockville, MD, USA) was used to calculate the myocardial collagen volume fraction.

ELISA. An ELISA double-antibody sandwich assay kit for Ang (1-7) was purchased from Cloud-Clone Corp Company (cat. no. CES085Ra; Katy, TX, USA). According to the kit instructions, the reaction system and standard curve were established. The absorbance of each sample was measured by enzyme-linked immunosorbent analyzer. The content of Ang (1-7) was calculated in accordance with the standard curve.

Immunohistochemical determination of myocardial ACE2, Ang (1-7), MasR and Ang II. 4\% paraformaldehyde-fixed, paraffin-embedded cardiac tissue sections were used for immunohistochemical staining. Following deparaffinization and hydration, the slides were washed in PBS. Endogenous peroxidase activity was quenched by incubating the slides in $3 \% \mathrm{H}_{2} \mathrm{O}_{2}$ for $10 \mathrm{~min}$. For antigen retrieval, the sections were treated with sodium citrate buffer for $20 \mathrm{~min}$ at $37^{\circ} \mathrm{C}$. Following overnight incubation with the primary antibodies [rabbit polyclonal anti-ACE2 antibody (cat. no. ab15348; Abcam, Cambridge, MA, USA; dilution, 1:200); rabbit polyclonal anti-Ang (1-7) antibody (cat. no. PAS085Ra01; Cloud-Clone Corp; dilution, 1:200); rabbit polyclonal anti-Ang II antibody (cat. no. 15348; Abcam; dilution, 1:200); rabbit polyclonal anti-Mas receptor antibody (cat. no. sc-135063; Santa Cruz Biotechnology, Inc., Dallas, TX, USA; dilution, 1:200)] at $4^{\circ} \mathrm{C}$, the slides were washed in PBS, then a goat anti-rabbit horseradish peroxidase-conjugated secondary antibody (cat. no. sc-2007; Santa Cruz Biotechnology, Inc.; dilution, 1:1,000) was added, and the slides were further incubated at room temperature for $30 \mathrm{~min}$. The color was developed with $0.05 \%$ 3,3'-diaminobenzidine tetrahydrochloride and $0.01 \% \mathrm{H}_{2} \mathrm{O}_{2}$, and counterstained with hematoxylin. A negative control without primary antibody was included in the experiment to ensure the antibody specificity. Myocardial immunoreactivity for ACE2, Ang (1-7), Mas receptor and Ang II was performed in 20 randomly selected fields of heart sections at x400 magnification by light microscopy.

$R N A$ preparation and reverse transcription-quantitative polymerase chain reaction $(R T-q P C R)$. Total RNA was 
prepared from rat cardiac tissue using TRIzol reagent (Roche Diagnostics, Basel, Switzerland). A total of $1 \mu \mathrm{g}$ RNA was reverse transcribed using the cDNA Synthesis kit (THUNDERBIRD SYBR qPCR Mix, Toyobo Co., Ltd., Osaka, Japan). The primers used in the present study were as follows: ACE2, MasR, Ang II (Shanghai Sangon Pharmaceutical Co., Ltd., Shanghai, China). The primer sequences are listed in Table I. RT-qPCR assays was performed using a miScript SYBR $^{\circledR}$ Green PCR kit (Qiagen GmbH, Hilden, Germany) and a ABI 7500 Real-Time PCR System (Applied Biosystems; Thermo Fisher Scientific, Inc., Waltham, MA, USA), according to the manufacturer's instructions. PCR reactions were performed at $95^{\circ} \mathrm{C}$ for $10 \mathrm{~min}$, followed by 40 cycles of $95^{\circ} \mathrm{C}$ for $15 \mathrm{sec}$ and $60^{\circ} \mathrm{C}$ for $1 \mathrm{~min}$. Densitometric analysis of PCR was performed using the Digital Imaging System (Bio-Rad Laboratories, Inc., Hercules, CA, USA). The use of equal amounts of mRNA in the RT-qPCR assays was confirmed by analyzing the expression levels of house-keeping gene $\beta$-actin. The results were calculated using the $2^{\Delta \Delta \mathrm{Cq}}$ method (17).

Western blot analysis. Myocardial protein expression was detected by western blotting. The bicinchoninic acid (BCA) method was used to detect protein concentration using an Enhanced BCA Protein Assay kit (Beyotime Institute of Biotechnology, Haimen, China), according to the manufacturer's instructions. Heart protein samples $(50 \mu \mathrm{g})$ were subjected to $10 \%$ SDS-PAGE, transferred to nitrocellulose membrane, and immunoblotted using ACE2 antibodies (Abcam; dilution, 1:1,000), MasR antibodies (Santa Cruz Biotechnology, Inc.; 1:1,000) and Ang II antibodies (Abcam; dilution, 1:1,000), Collagen I antibodies (Abcam; dilution, 1:1,000), Collagen III antibodies (Abcam; dilution, 1:2,000), $\beta$-actin (Abcam; dilution, 1:1,000) was used as loading control to determine the relative expression levels of the target protein in the sample. Western blotting bands of the target protein were analyzed; the results are presented as the ratio of integrated optical density, and statistical analysis was conducted.

Statistical analysis. Experimental data are shown as mean \pm standard deviation. Main and interactive effects were analyzed by one-way analysis of variance (ANOVA) using SPSS software (version, 22.0; IBM SPSS, Armonk, NY, USA). When justified by one-way ANOVA, differences between individual group means were analyzed by Fisher's LSD test. $\mathrm{P}<0.05$ was considered to indicate a statistically significant difference.

\section{Results}

Cardiac remodeling and left ventricle dysfunction in post-myocardial infarction. Compared with the rats in the sham group, the left ventricular remodeling progression increased [left ventricular mass (LVM), left ventricular internal end-systolic diameter (LVIDs), left ventricular internal end-diastolic diameter indicators increased (LVIDd); $\mathrm{P}<0.05]$, and systolic function abnormalities started to appear [left ventricular ejection fraction $(\mathrm{EF})$ decreased; $\mathrm{P}<0.05]$. The left ventricular mass, the systolic function abnormalities, the left ventricular internal end-systolic diameter and left ventricular internal end-diastolic diameter are improved in the Tel and Olm treated groups when compared with the MI group $(\mathrm{P}<0.05)$, however, there is no significant difference between the two groups. However, Olm could not completely improve the cardiac function state and restore it to normal values (compared with the sham group on LVIDs, LVIDd, LVM and EF, P<0.05; Table II).

Collagen deposition in post-myocardial infarction. Collagen deposition leads to myocardial fibrosis, which can result in myocardial remodeling. The degree of collagen deposition in the post-myocardial infarction rats could be identified using Masson staining (Fig. 1A). Normal myocardial tissue was red, and the blue fibrin component appeared in the myocardial interstitium in myocardial infarction group. Collagen deposition induced myocardial interstitial fibrosis could limit myocardial diastolic function. The volume fraction of myocardial collagen was calculated using Image-Pro Plus software. The collagen volume fraction of the MI group increased significantly compared with that of the sham group [Sham (3.85 \pm 0.60$)$; MI (11.14 \pm 1.48$), \mathrm{P}<0.05$; Fig. 1B]. However, Tel and Olm significantly reduced the degree of myocardial fibrosis in post-myocardial infarction rats; the effect of Olm is more marked [Tel (8.81 \pm 0.6$)$; Olm (7.6 \pm 0.69$), \mathrm{P}<0.05$; Fig. 1B].

Ang (1-7) and Ang II level. The expression of Ang II in the plasma of the MI group increased compared with that of the sham group $(\mathrm{P}<0.05$; Fig. $2 \mathrm{~A})$, meanwhile the expressions of Ang (1-7) increased ( $\mathrm{P}>0.05$; Fig. 2B). Following intervention with Tel and Olm, compared with the MI group, the content of plasma Ang (1-7) significantly increased in the Tel group ( $\mathrm{P}<0.05$; Fig. 2B), whereas Ang II expression decreased in the Tel group ( $\mathrm{P}<0.05$; Fig. $2 \mathrm{~A})$, and a more obvious effect was observed in the Olm group ( $\mathrm{P}<0.01$; Fig. $2 \mathrm{~A}$ and $\mathrm{B})$.

Immunohistochemical determination of myocardial ACE-2, Ang (1-7), MasR and Ang II. In sham rats, the expressions of ACE2, Ang (1-7), MasR and Ang II occurred in cardiomyocytes (Fig. 3A-D). Myocardial infarction increased all immune staining simultaneously, while the expressions of Ang II increased significantly $(\mathrm{P}<0.05)$. Following intervention with Tel or Olm, compared with the MI group, the expression of AngIIdecreased, conversely ACE2, Ang (1-7) and MasR expression increased $(\mathrm{P}<0.05)$, and the effect of the Olm group is more marked than that of the Tel group $(\mathrm{P}<0.05)$.

Protein expression of ACE2, MasR, Ang II, Collagen I and CollagenIII in myocardial tissue. To investigate the protein expression of ACE2, MasR, Ang II, Collagen I and Collagen III, the authors extracted the total protein from myocardial tissue. Consistent with mRNA level, Ang II, Collagen I and Collagen III protein expression significantly increased in the MI group compared with sham group following surgery for 4 weeks $(\mathrm{P}<0.05$; Fig. 4A, D and E). ACE2 and MasR protein expression increased slightly in the MI group (Fig. 4B and C). Following treatment with Tel and Olm, Ang II, Collagen I and Collagen III protein expression notably decreased, meanwhile, ACE2 and MasR protein expression increased, and more obviously in the Olm group $(\mathrm{P}<0.05$; Fig. 4A-E).

The mRNA expression of ACE2, MasR and Ang II in myocardial tissue. To test mRNA level of ACE2-Ang (1-7)-Mas, the 
Table I. Specific primers for reverse transcription-quantitative polymerase chain reaction.

\begin{tabular}{lll}
\hline Gene name & \multicolumn{1}{c}{ Primer sequence (forward) } & \multicolumn{1}{c}{ Primer sequence (reverse) } \\
\hline Ang II & 5'-TTGGGTGCTGAGGCAAATCT-3' & 5'-TTGGGTGCTGAGGCAAATCT-3' \\
ACE2 & 5'-GTGGAGCACTGACTGGAGC-3' & 5'-GACAGGAGGCTCGTAAGGTG-3' \\
MasR & 5'-TGACAGCCATCAGTGTGGAGA-3' & 5'-GCATGAAAGTGCCCACAGGA-3' \\
$\beta$-actin & 5'-CCCTGTGCTGCTCACCGA-3' & 5'-ACAGTGTGGGTGACCCCGTC-3'
\end{tabular}

Ang, angiotensin; ACE2, angiotensin-converting enzyme 2; MasR, Mas receptor.

Table II. Cardiac function test of post-myocardial infarction rats.

\begin{tabular}{lcrrr}
\hline & Sham $(\mathrm{n}=10)$ & MI $(\mathrm{n}=10)$ & Tel $(\mathrm{n}=10)$ & Olm $(\mathrm{n}=10)$ \\
\hline BW (g) & $339.98 \pm 21.61$ & $328.54 \pm 12.63$ & $342.45 \pm 25.10$ & $336.05 \pm 18.91$ \\
HW (mg) & $1.00 \pm 0.23$ & $1.25 \pm 0.12^{\mathrm{a}}$ & $1.12 \pm 0.09^{\mathrm{a}, \mathrm{b}}$ & $1.12 \pm 0.08^{\mathrm{a}, \mathrm{b}}$ \\
LVM (mg) & $0.81 \pm 0.34$ & $1.08 \pm 0.10^{\mathrm{a}}$ & $0.96 \pm 0.08^{\mathrm{a}, \mathrm{b}}$ & $0.99 \pm 0.05^{\mathrm{a}, \mathrm{b}}$ \\
LV/BW (mg/g) & $2.38 \pm 0.17$ & $3.28 \pm 0.33^{\mathrm{a}}$ & $2.82 \pm 0.35^{\mathrm{a}, \mathrm{b}}$ & $2.95 \pm 0.18^{\mathrm{a}, \mathrm{b}}$ \\
LVIDs (mm) & $3.47 \pm 0.82$ & $6.38 \pm 0.66^{\mathrm{a}}$ & $5.01 \pm 0.78^{\mathrm{a}, \mathrm{b}}$ & $4.49 \pm 0.79^{\mathrm{a}, \mathrm{b}}$ \\
LVIDd (mm) & $6.11 \pm 0.38$ & $8.34 \pm 0.78^{\mathrm{a}}$ & $7.49 \pm 0.61^{\mathrm{a}, \mathrm{b}}$ & $7.09 \pm 0.45^{\mathrm{a}, \mathrm{b}}$ \\
EF (\%) & $84.27 \pm 4.69$ & $52.14 \pm 5.47^{\mathrm{a}}$ & $57.98 \pm 6.04^{\mathrm{a}, \mathrm{b}}$ & $65.78 \pm 7.79^{\mathrm{a}, \mathrm{b}, \mathrm{c}}$
\end{tabular}

${ }^{\mathrm{a}} \mathrm{P}<0.05$ vs. sham group; ${ }^{\mathrm{b}} \mathrm{P}<0.05$ vs. MI group; ${ }^{\mathrm{C}} \mathrm{P}<0.05$ vs. Tel group ( $\mathrm{n}=10 /$ group). $\mathrm{BW}$, body weight; HW, heart weight; $\mathrm{LVM}$, left ventricular mass; LVIDs, left ventricular internal end-systolic diameter; LVIDd, left ventricular internal end-diastolic diameter indicators increased; EF, left ventricular ejection fraction; MI, myocardial infarction; Tel, telmisartan (5 mg/kg); Olm, olmesartan (5 mg/kg).

authors extracted RNA from myocardial tissue and conducted RT-qPCR. The results indicated that ACE2 and MasR mRNA expression are slightly increased in the MI group $(\mathrm{P}<0.05$; Fig. 5A, C and D). Following treatment with Tel and Olm, the expression of ACE2 and MasR mRNA was increased $(\mathrm{P}<0.05$; Fig. 5A, C and D). Compared with the sham group, Ang II mRNA level significantly increased in the MI group $(\mathrm{P}<0.05)$, while its expression decreased following drug-treatment intervention (Fig. 5A and B). The effect of Olm is more significantly than Tel ( $\mathrm{P}<0.05$; Fig. 5A-D).

\section{Discussion}

Inhibiting the RAS is an important strategy for cardioprotection, not only by controlling blood pressure, but also by reducing target-organ damage. Indeed, treatment with RAS blockers showed clear benefits in distinct stages of cardiovascular disease. These treatments reduce cardiovascular events in patients with subclinical lesions, and prolong survival in patients with clinical disease or organ dysfunction $(2,18)$.

The left ventricular remodeling progression increased in the rat of myocardial infarction by ligating the left coronary artery, the expression of Ang II, ACE2, MasR and collagen deposition increased more than sham group. Treatment with Tel and Olm could improve cardiac function, alleviate collagen deposition, antagonize Ang II and increase ACE2, MasR, Ang (1-7) expression in myocardial tissue, and the effect of Olm was more remarkable than that of Tel. These findings indicated that $\mathrm{Olm}$ treatment inhibits cardiac remodeling and fibrosis not only through its original Ang II receptor 1 (AT1R) blockade, but partly through enhancement of the ACE2-Ang (1-7)-MasR axis pathway.

As expected, myocardial infarction involves cardiac dysfunction and cardiac remodeling caused by an increase in Ang II through the AT1R signaling. Consistent with this, the present study demonstrated that cardiac dysfunction and cardiac remodeling occurred in the MI group, as demonstrated by echocardiography and histological assessments. Consistent with these results, the authors demonstrated that the expression of Ang II was higher in the MI group, and that the decrease of Ang II was induced by treatment with Ang II receptor blockers (ARBs) Tel and Olm. Olm was more effective at reducing Ang II levels. Furthermore, these results are supported by the previous report, stating that Olm can downregulate Ang II levels both in cardiomyocytes in vivo and in vitro $(19,20)$.

The extracellular matrix (ECM) is a living network of proteins that maintains the structural integrity of the myocardium, collagen is an important mediator for cardiac fibrosis induced by Ang II, so cardiac remodeling and fibrosis are closely linked each other $(21,22)$. Lindsey et al (23) reported that collagen $\mathrm{I} \alpha 1$ matricryptin p1158/59 facilitates LV remodeling in post-MI by regulating scar formation through targeted ECM generation and stimulation of angiogenesis. In the present study, the expression of collagen genes was increased in the MI group, while the increase observed in the ARB-drug group was inhibited by Olm or Tel treatment. Under the same concentration $(5 \mathrm{mg} / \mathrm{kg})$, the effect of $\mathrm{Olm}$ 
A

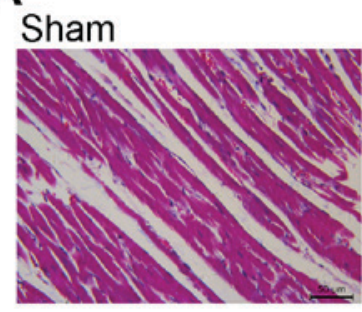

Tel

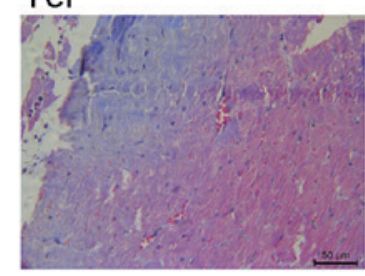

MI

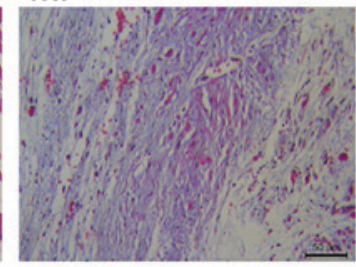

Olm

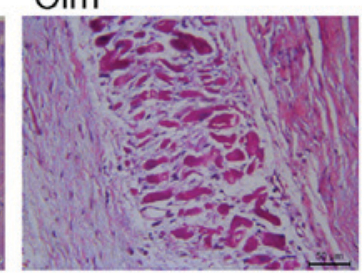

B

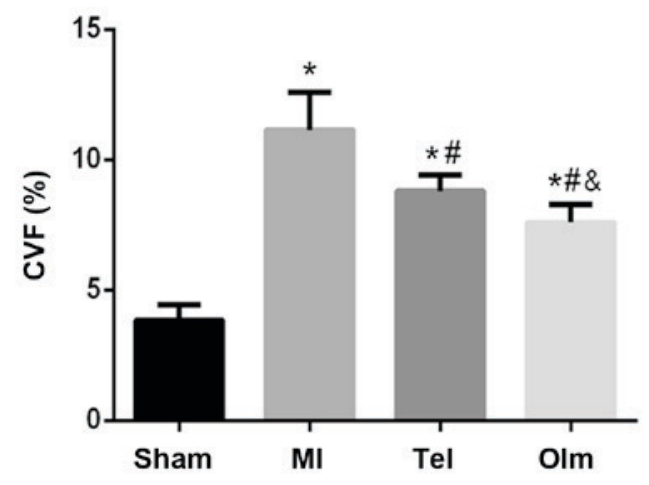

Figure 1. Collagen deposition in post-myocardial infarction. (A) Myocardial Masson staining of each group. Normal myocardium is presented in red, and cellulose is presented in blue (magnification, $\mathrm{x} 400$ ). (B) CVF in sham, MI, Tel and Olm groups. $\mathrm{P}<0.05 \mathrm{vs}$. sham group; ${ }^{\star} \mathrm{P}<0.05 \mathrm{vs}$. Tel group; ${ }^{*} \mathrm{P}<0.05 \mathrm{vs}$. MI group. ( $\mathrm{n}=10$ each group). CVF, collagen volume fraction; MI, myocardial infarction; Tel, telmisartan (5 mg/kg); Olm, olmesartan (5 mg/kg); CVF, collagen volume fraction.

A

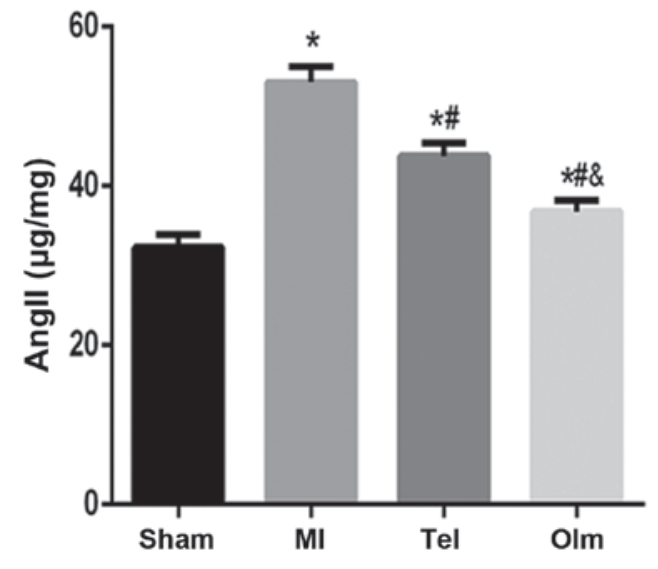

B

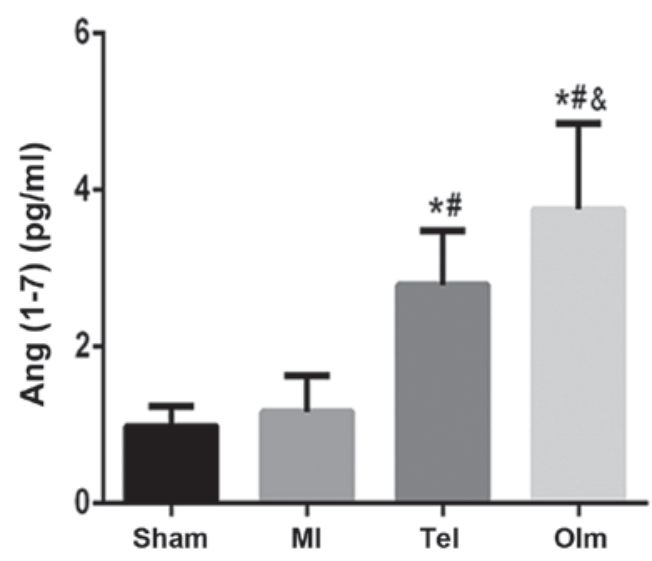

Figure 2. Ang (1-7) and Ang II level. (A) The plasma level of Ang II. (B) The plasma level of Ang (1-7). ${ }^{*} \mathrm{P}<0.05$ vs. sham group; ${ }^{*} \mathrm{P}<0.05$ vs. MI group; ${ }^{\text {P }}<0.05$ vs. Tel group. Ang, angiotensin.

is more interesting. These findings suggested that Olm can block collagen expression and deposition via reducing Ang II levels.

Accumulating evidence suggests that the ACE2-Ang (1-7)-Mas pathway has a crucial role in cardio protection (24). ACE2 gene expression is upregulated in the infarct zone and survival myocardial tissue from mice with myocardial infarction (25). Consistent with the previous studies, these data demonstrated that the mRNA and protein expression of ACE2 in myocardial tissues was increased slightly in MI rats, while administration of ARB significantly upregulated the expression of ACE2 to protect the heart. It has been reported that blockage of RAS by an ACE inhibitor or ARB increases cardiac Ang (1-7), ACE2 mRNA and ACE2 activity. Overexpression of ACE2 can attenuate ventricular fibrosis and improved ventricular remodeling in a rat model of diabetic cardiomyopathy (26). ACE2 deficiency worsened epicardial adipose tissue inflammation and cardiac dysfunction in response to diet-induced obesity (27). Clinical studies have also demonstrated that expression of the
ACE2 gene had a positive correlation with cardiac remodeling. Serum ACE2 activity rises in relation to infarct size, left ventricular systolic dysfunction and is associated with the occurrence of left ventricular remodeling in patient with myocardial infarction (28). Increased ACE/ACE2 ratios may induce Ang II overactivation and accelerate cardiac remodeling in patients with moderate to severe heart failure (29). In the present study, the cardiac remodeling and fibrosis were attenuated by ARB treatment, and also associated with an ACE2 increase. Moreover, the increase of ACE2 in the Olm group was more remarkable than in the Tel group. In addition, ACE2 overexpression significantly inhibits the progression of atherosclerosis (30), oxidative stress and inflammatory cytokine expression (31), SNPs in the ACE2 gene are also associated with $\mathrm{LVH}$, impaired cardiac function and HT $(32,33)$. Therefore, the ACE2 gene is considered as a functional candidate for CVD (34), and also a potential therapy target of Olm.

Still, Ang (1-7) is a cardiovascular protective peptide in RAS against cardiovascular toxic effects of Ang II. Like other 
A

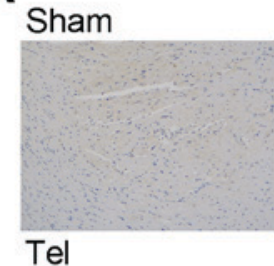

Tel

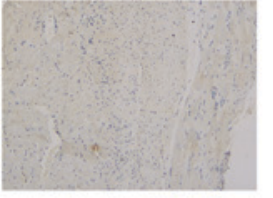

B

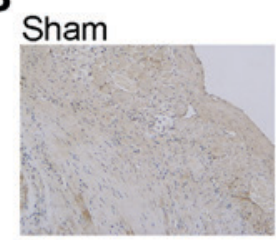

Tel

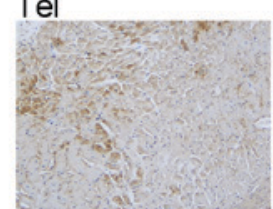

C

\section{Sham}

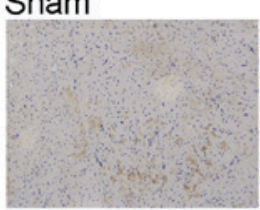

Tel

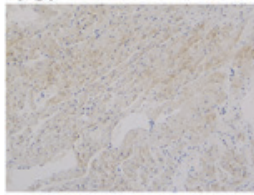

$\mathbf{D}_{\text {Sham }}$

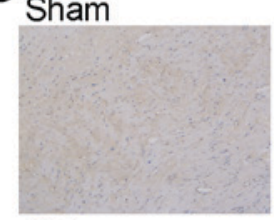

Tel

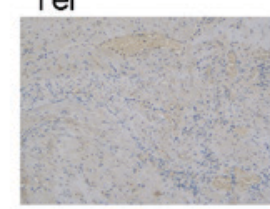

$\mathrm{Ml}$

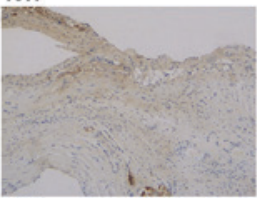

Olm

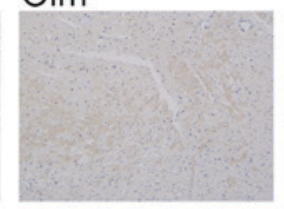

MI

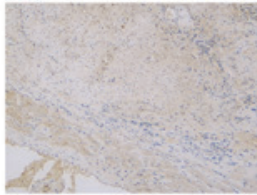

Olm

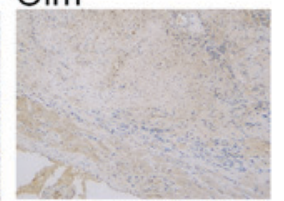

MI

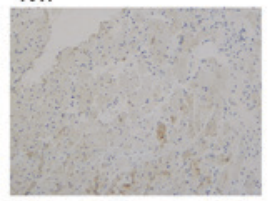

Olm

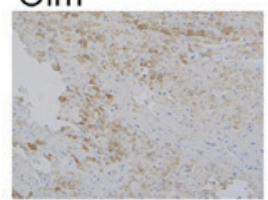

MI

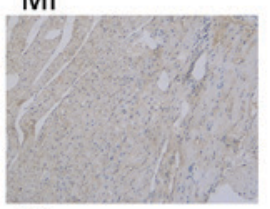

Olm

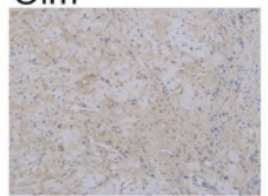

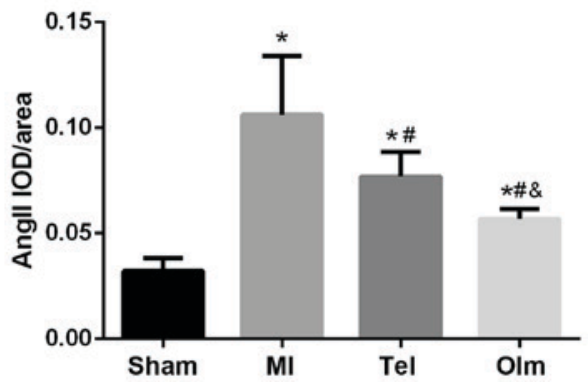
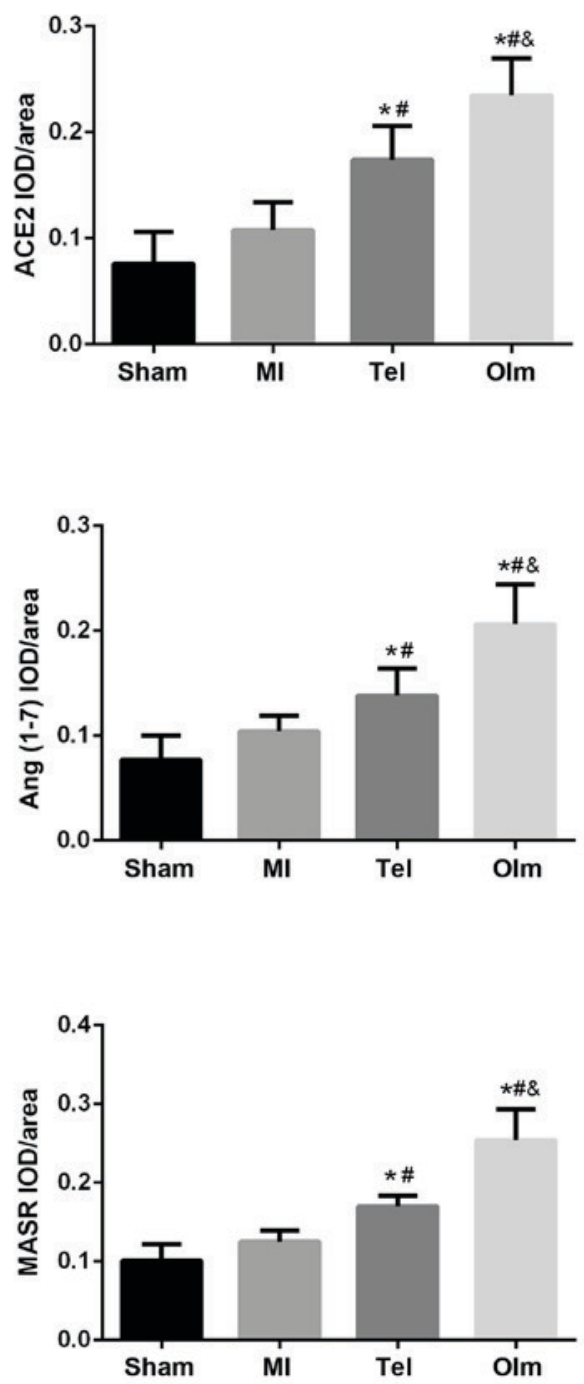

Figure 3. Immunohistochemical determination of myocardial ACE-2, Ang (1-7), MasR and Ang II. (A) Light microscopic images of Ang II immunohistochemical labeling (brown staining) of cardiomyocytes in left ventricle. (B) Light microscopic images of ACE2 immunohistochemical labeling (brown staining) of cardiomyocytes in left ventricle. (C) Light microscopic images of Ang (1-7) immunohistochemical labeling (brown staining) of cardiomyocytes in left ventricle. (D) Light microscopic images of MasR immunohistochemical labeling (brown staining) of cardiomyocytes in left ventricle. Scale bar = $200 \mu \mathrm{m}$; magnification, $\mathrm{x} 100$. Data are expressed as means \pm standard error of the mean. ${ }^{\mathrm{P}}<0.05$ vs. sham group; ${ }^{*} \mathrm{P}<0.05$ vs. MI group; ${ }^{\circledR} \mathrm{P}<0.05 \mathrm{vs}$. Tel group $(\mathrm{n}=10$ each group). ACE2, angiotensin-converting enzyme 2; Ang, angiotensin; MasR, Mas receptor; MI, myocardial infarction; Tel, telmisartan (5 mg/kg); Olm, olmesartan $(5 \mathrm{mg} / \mathrm{kg})$.

peptides, Ang (1-7) has a physiological role when it binds to the specific MasR. MasR is involved in the homeostasis of the heart, as well as in the establishment and progression of cardiac diseases (35). There is accumulating interest in viewing 
A
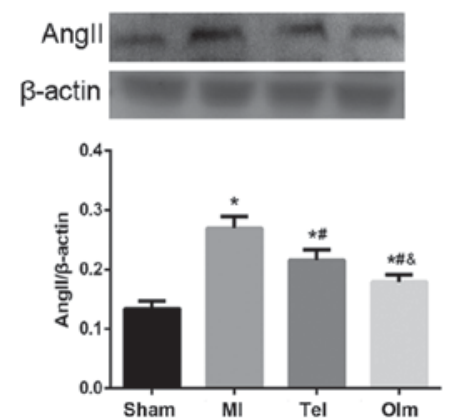

D
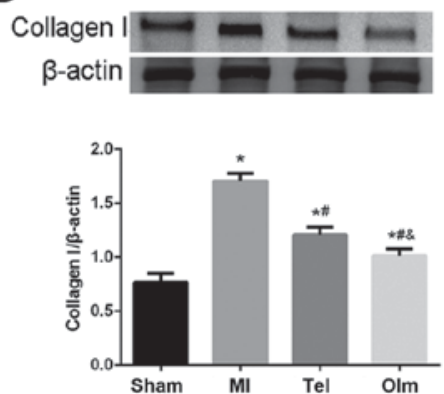

B
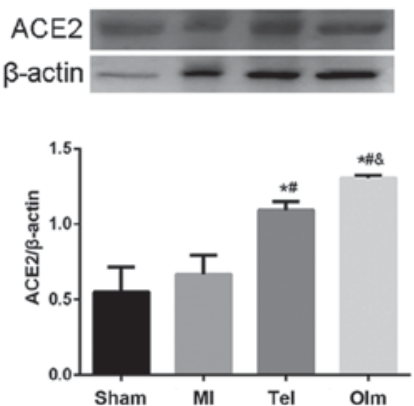

E
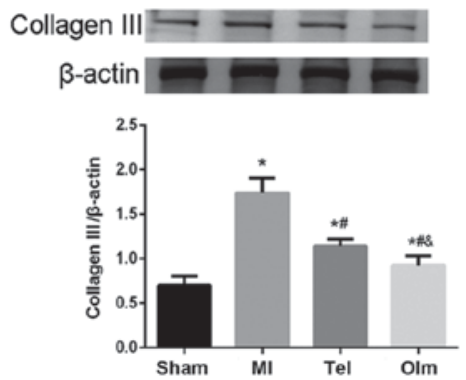

C
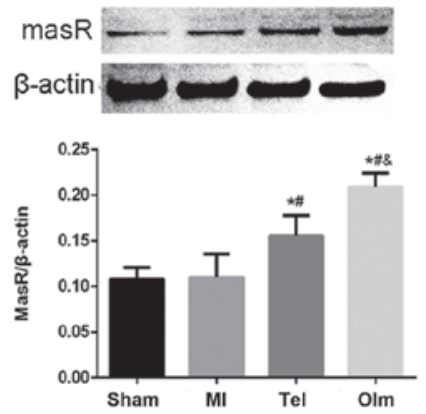

Figure 4. The protein expression of ACE2, MasR, Ang II Collagen I and Collagen III in myocardial tissue. Myocardial tissue homogenates from MI group or Tel treatment were subjected to SDS-PAGE. The non-responsive gene $\beta$-actin is shown as loading control. (A) Quantitative analysis of Ang II protein expression. (B) Quantitative analysis of ACE2 protein expression. (C) Quantitative analysis of Mas protein expression. (D) Quantitative analysis of Collagen I protein expression. (E) Quantitative analysis of Collagen III protein expression. " $\mathrm{P}<0.05$ vs. sham group. ${ }^{\#} \mathrm{P}<0.05$ vs. MI group; ${ }^{\&} \mathrm{P}<0.05$ vs. Tel group $(\mathrm{n}=10$ each group). ACE2, angiotensin-converting enzyme 2; MasR, Mas receptor; Ang, angiotensin; Tel, telmisartan (5 mg/kg); Olm, olmesartan (5 mg/kg).

A

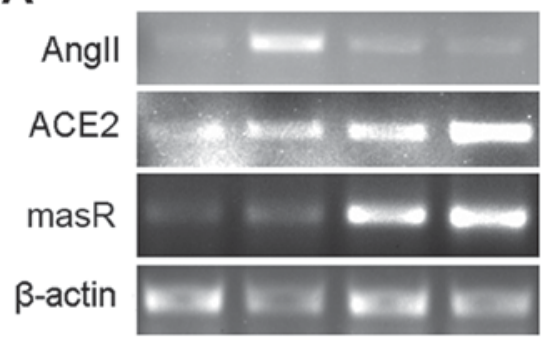

C

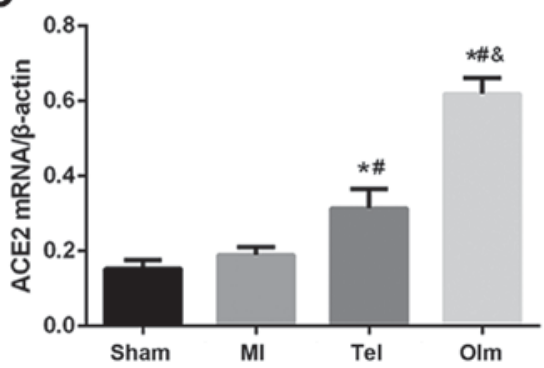

B

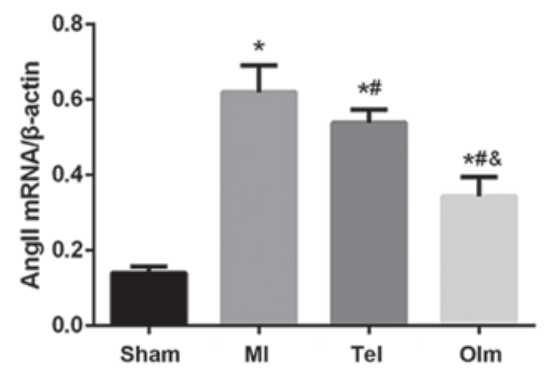

D

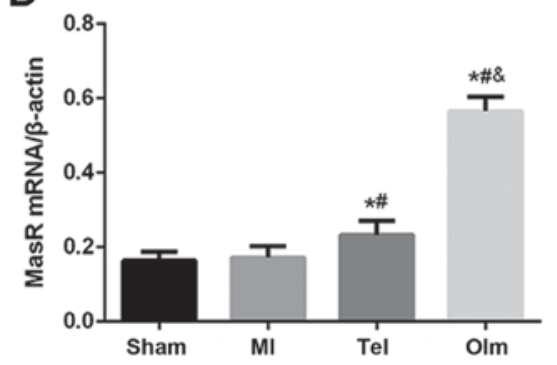

Figure 5. The mRNA expression of ACE2, MasR and Ang II in myocardial tissue. (A and C) Cardiac ACE2 mRNA, (A and D) MasR mRNA and (A and B) Ang II mRNA. (A) sham operation group; (B) MI group; (C) Tel group (5 mg/kg); (D) Olm group (5 mg/kg). ${ }^{*} \mathrm{P}<0.05 \mathrm{vs}$. sham group; ${ }^{\sharp} \mathrm{P}<0.05 \mathrm{vs}$. MI group; ${ }^{\&} \mathrm{P}<0.05$ vs. Tel group ( $\mathrm{n}=10$ each group). ACE2, angiotensin-converting enzyme 2; MasR, Mas receptor; Ang, angiotensin; Tel, telmisartan (5 mg/kg); Olm, olmesartan (5 mg/kg).

MasR as a crucial modulator and pharmacological target in cardiovascular disease research (36). Studies have confirmed that Ang (1-7) reduces the growth of cardiomyocytes through activation of the MasR (37). Ang (1-7) promotes angiogenesis via stimulating the expression of cardiac vascular endothelial growth factor-D and matrix metalloproteinase-9, thus 
facilitating cardiac repair and ventricular function (38). The cardioprotective effects of Ang (1-7) are mediated by MasR to different signaling pathways involving mitogen-associated protein kinase, phosphoinositide3-kinase/protein kinase B and NADPH oxidase $(15,16)$. Consistently, the current study demonstrated that the expression of Ang (1-7) and MasR was slightly increased in the MI group, while upregulated following Tel and Ole intervention, suggesting a protective role of MasR in the development of the heart.

In conclusion, the current study presents that the ACE2-Ang (1-7)-MasR axis is closely related with the development of MI-induced cardiac remodeling of rats. The levels of ACE2 and Ang (1-7) increased both in plasma and myocardial tissue from MI group, as well as MasR level. Under the same concentrations of Olm and Tel, Olm was more effective on modulating the activities of components of the Ang (1-7)-ACE2-MasR axis in plasma and myocardial tissue. Their expression levels were significantly upregulated when treated with Olm. Moreover, Olm reduced the collagen deposition, delay the left ventricular remodeling and eventually improved the cardiac function in MI rats. Taken together, Olm can inhibit myocardial fibrosis of MI rats through activating the ACE2-Ang (1-7)-MasR signaling pathway. Research on the contribution of the ACE2-Ang (1-7)-MasR axis to cardiovascular disease will lead to the development of new pharmacological approaches resulting in the design of molecular or genetic means to targeting modulate the expression of ACE2, Ang (1-7) or MasR, which will provide new targets for drug design, drug combination therapy and gene therapy for cardiovascular diseases.

\section{Acknowledgements}

The present work was partly supported by funds of research projects of the Xinjiang Uyghur Autonomous Region Project of Science and Technology (grant no. 2016E02075) and the National Natural Science Foundation (grant no. 81560689).

\section{References}

1. Mendis S, Davis S and Norrving B: Organizational update: The world health organization global status report on noncommunicable diseases 2014; one more landmark step in the combat against stroke and vascular disease. Stroke 46: e121-e122, 2015.

2. Houston Miller N: Cardiovascular risk reduction with Renin-Angiotensin aldosterone system blockade. Nurs Res Pract 2010: 101749, 2010.

3. Schmieder RE, Hilgers KF, Schlaich MP and Schmidt BM: Renin-angiotensin system and cardiovascular risk. Lancet 369: 1208-1219, 2007.

4. Ferrario CM and Strawn WB: Role of the renin-angiotensin-aldosterone system and proinflammatory mediators in cardiovascular disease. Am J Cardiol 98: 121-128, 2006.

5. Mehta PK and Griendling KK: Angiotensin II cell signaling: Physiological and pathological effects in the cardiovascular system. Am J Physiol Cell Physiol 292: C82-C97, 2007.

6. Chappell MC: Nonclassical renin-angiotensin system and renal function. Compr Physiol 2: 2733-2752, 2012.

7. McKinney CA, Fattah C, Loughrey CM, Milligan G and Nicklin SA: Angiotensin-(1-7) and angiotensin-(1-9): Function in cardiac and vascular remodelling. Clin Sci (Lond) 126: 815-827, 2014.

8. Varagic J, Ahmad S, Nagata S and Ferrario CM: ACE2: Angiotensin II/angiotensin-(1-7) balance in cardiac and renal injury. Curr Hypertens Rep 16: 420, 2014.

9. Ocaranza MP, Michea L, Chiong M, Lagos CF, Lavandero S and Jalil JE: Recent insights and therapeutic perspectives of angiotensin-(1-9) in the cardiovascular system. Clin Sci (Lond) 127: 549-557, 2014.
10. Moritani T, Iwai M, Kanno H, Nakaoka H, Iwanami J, Higaki T, Ishii E and Horiuchi M: ACE2 deficiency induced perivascular fibrosis and cardiac hypertrophy during postnatal development in mice. J Am Soc Hypertens 7: 259-266, 2013.

11. Iwanami J, Mogi M, Tsukuda K, Wang XL, Nakaoka H, Ohshima K, Chisaka T, Bai HY, Kanno H, Min LJ and Horiuchi M: Role of angiotensin-converting enzyme 2/angiotensin-(1-7)/Mas axis in the hypotensive effect of azilsartan. Hypertens Res 37: 616-620, 2014.

12. Zhang Z, Chen L, Zhong J, Gao P and Oudit GY: ACE2/Ang-(1-7) signaling and vascular remodeling. Sci China Life Sci 57: 802-808, 2014.

13. Zhang Y, Li B, Wang B, Zhang J, Wu J and Morgan T: Alteration of cardiac ACE2/Mas expression and cardiac remodelling in rats with aortic constriction. Chin J Physiol 57: 335-342, 2014.

14. Liang B, Li Y, Han Z, Xue J, Zhang Y, Jia S and Wang C: ACE2-Ang (1-7) axis is induced in pressure overloaded rat model. Int J Clin Exp Pathol 8: 1443-1450, 2015.

15. Nemoto W, Ogata Y, Nakagawasai O, Yaoita F, Tadano T and Tan-No K: Angiotensin (1-7) prevents angiotensin II-induced nociceptive behaviour via inhibition of p38 MAPK phosphorylation mediated through spinal Mas receptors in mice. Eur J Pain 18: 1471-1479, 2014.

16. Zheng J,Li G, Chen S, Bihl J, Buck J,Zhu Y, Xia H, Lazartigues E, Chen Y and Olson JE: Activation of the ACE2/Ang-(1-7)/Mas pathway reduces oxygen-glucose deprivation-induced tissue swelling, ROS production, and cell death in mouse brain with angiotensin II overproduction. Neuroscience 273: 39-51, 2014.

17. Livak KJ and Schmittgen TD: Analysis of relative gene expression data using real-time quantitative PCR and the 2(-Delta Delta C(T)) method. Methods 25: 402-408, 2001.

18. de la Sierra A: Renin-angiotensin system blockade and reduction of cardiovascular risk: Future perspectives. Expert Rev Cardiovasc Ther 9: 1585-1591, 2011.

19. Wang X, Ye Y, Gong H, Wu J, Yuan J, Wang S, Yin P, Ding Z, Kang L, Jiang Q, et al: The effects of different angiotensin II type 1 receptor blockers on the regulation of the ACE-AngII-AT1 and ACE2-Ang(1-7)-Mas axes in pressure overload-induced cardiac remodeling in male mice. J Mol Cell Cardiol 97: 180-190, 2016.

20. Tanno T, Tomita H, Narita I, Kinjo T, Nishizaki K, Ichikawa H, Kimura Y, Tanaka M, Osanai T and Okumura K: Olmesartan inhibits cardiac hypertrophy in mice overexpressing renin independently of blood pressure: Its beneficial effects on ACE2/Ang(1-7)/Mas Axis and NADPH oxidase expression. J Cardiovasc Pharmacol 67: 503-509, 2016.

21. Segura AM, Frazier OH and Buja LM: Fibrosis and heart failure. Heart Fail Rev 19: 173-185, 2014.

22. Schnee JM and Hsueh WA: Angiotensin II, adhesion, and cardiac fibrosis. Cardiovasc Res 46: 264-268, 2000.

23. Lindsey ML, Iyer RP, Zamilpa R, Yabluchanskiy A, DeLeon-Pennell KY, Hall ME, Kaplan A, Zouein FA, Bratton D, Flynn ER, et al: A novel collagen matricryptin reduces left ventricular dilation post-myocardial infarction by promoting scar formation and angiogenesis. J Am Coll Cardiol 66: 1364-1374, 2015.

24. Chamsi-PashaMA,ShaoZ and Tang WH: Angiotensin-converting enzyme 2 as a therapeutic target for heart failure. Curr Heart Fail Rep 11: 58-63, 2014.

25. Burrell LM, Risvanis J, Kubota E, Dean RG, MacDonald PS, Lu S, Tikellis C, Grant SL, Lew RA, Smith AI, et al: Myocardial infarction increases ACE2 expression in rat and humans. Eur Heart J 26: 369-375, 322-324, 2005.

26. Dong B, Yu QT, Dai HY, Gao YY, Zhou ZL, Zhang L, Jiang H, Gao F, Li SY, Zhang YH, et al: Angiotensin-converting enzyme-2 overexpression improves left ventricular remodeling and function in a rat model of diabetic cardiomyopathy. J Am Coll Cardiol 59: 739-747, 2012.

27. Montaigne D, Coisne A, Marechal X and Staels B: Comment on Patel et al: ACE2 deficiency worsens epicardial adipose tissue inflammation and cardiac dysfunction in response to diet-induced obesity. Diabetes 2016;65:85-95. Diabetes 65: e1-e2, 2016.

28. Ortiz-Pérez JT, Riera M, Bosch X, De Caralt TM, Perea RJ, Pascual $J$ and Soler MJ: Role of circulating angiotensin converting enzyme 2 in left ventricular remodeling following myocardial infarction: A prospective controlled study. PLoS One 8: e61695, 2013

29. Wang J, Li N, Gao F, Song R, Zhu S and Geng Z: Balance between angiotensin converting enzyme and angiotensin converting enzyme 2 in patients with chronic heart failure. J Renin Angiotensin Aldosterone Syst 16: 553-558, 2015. 
30. Wang Y, Tikellis C, Thomas MC and Golledge J: Angiotensin converting enzyme 2 and atherosclerosis. Atherosclerosis 226: 3-8, 2013.

31. Simões e Silva AC, Silveira KD, Ferreira AJ and Teixeira MM: ACE2, angiotensin-(1-7) and Mas receptor axis in inflammation and fibrosis. Br J Pharmacol 169: 477-492, 2013.

32. Yang M, Zhao J, Xing L and Shi L: The association between angiotensin-converting enzyme 2 polymorphisms and essential hypertension risk: A meta-analysis involving 14,122 patients. J Renin Angiotensin Aldosterone Syst 16 : 1240-1244, 2015.

33. Wang SX, Tao T, Fu ZQ, Xie XZ, Wang H and Wang YT: Polymorphisms of angiotensin-converting enzyme 2 gene confer a risk to lone atrial fibrillation in Chinese male patients. Chin Med J (Engl) 126: 4608-4611, 2013.

34. Burrell LM, Harrap SB, Velkoska E and Patel SK: The ACE2 gene: Its potential as a functional candidate for cardiovascular disease. Clin Sci (Lond) 124: 65-76, 2013.
35. Lin L, Liu X, Xu J, Weng L, Ren J, Ge J and Zou Y: Mas receptor mediates cardioprotection of angiotensin-(1-7) against Angiotensin II-induced cardiomyocyte autophagy and cardiac remodelling through inhibition of oxidative stress. J Cell Mol Med 20: 48-57, 2016

36. Singh K, Sharma K, Singh M and Sharma PL: Possible mechanism of the cardio-renal protective effects of AVE-0991, a non-peptide Mas-receptor agonist, in diabetic rats. J Renin Angiotensin Aldosterone Syst 13: 334-340, 2012.

37. Tallant EA, Ferrario CM and Gallagher PE: Angiotensin-(1-7) inhibits growth of cardiac myocytes through activation of the mas receptor. Am J Physiol Heart Circ Physiol 289: H1560-H1566, 2005.

38. Zhao W, Zhao T, Chen Y and Sun Y: Angiotensin 1-7 promotes cardiac angiogenesis following infarction. Curr Vasc Pharmacol 13: 37-42, 2015. 